\title{
MDAnderson CancerCenter \\ A challenging case of new-onset paroxysmal atrial fibrillation with rapid ventricular response secondary to autoimmune thyroiditis after a single dose of nivolumab-ipilimumab
}

\author{
Norman Brito-Dellan, M.D.
}

The University of Texas MD Anderson Cancer Center

Department of General Internal Medicine, Section of Hospital Medicine, Houston, Texas U.S.A.

A 61-year-old man with moderately differentiated distal esophageal adenocarcinoma, HER-2 2+ by IHC, HER-2 negative by FISH, metastatic to liver, left adrenal gland, and lymph nodes, was randomized to nivolumab $1 \mathrm{mg} / \mathrm{kg}(93 \mathrm{mg})$ and ipilimumab $3 \mathrm{mg} / \mathrm{kg}(280 \mathrm{mg})$ intravenously every 21 days. He was admitted to the hospital 19 days after initiation of therapy secondary to worsening dysphagia, inability to tolerate oral intake, early satiety, nausea, and vomiting. He underwent placement of a percutaneous gastrostomy tube the following day for nutritional needs. The next morning, 21 days after his first dose of the immune checkpoint inhibitors, he developed near syncope, lightheadedness, and diaphoresis after shaving his face. His heart rate exceeded 170 and was irregularly irregular (see Figure). This required vagal maneuvers, intravenous doses of metoprolol, digoxin, and initiation of a diltiazem intravenous drip for 24 hours, to achieve rate control and hemodynamic stability. The patient had no additional symptomatology, including no headaches, no visual disturbances, no polyuria, and no polydipsia. He has no history of hypertension, diabetes mellitus, or coronary arteriosclerosis.

An extensive work-up ensued, including serial cardiac enzymes, and EKGs that demonstrated no evidence of an acute coronary syndrome. A transthoracic echocardiogram showed a normal left ventricular ejection fraction (LVEF) of 70\%, with no regional wall motion abnormalities. A computed tomography angiography of the chest demonstrated no evidence of pulmonary embolism. Laboratory tests revealed normal electrolytes, including serum sodium and potassium, normal glycemia, and normal renal function tests. Additionally, we confirmed no evidence of hypophysitis or adrenalitis, with normal LH at $8.1 \mathrm{mIU} / \mathrm{mL}$, normal FSH at $4.6 \mathrm{mIU} / \mathrm{mL}$, normal IGF-1 at $35 \mathrm{ng} / \mathrm{mL}$, as well as an intact adrenal cortical axis with normal ACTH at $9 \mathrm{pg} / \mathrm{mL}$, normal morning cortisol levels at $16.5 \mathrm{mcg} / \mathrm{dL}$, normal renin activity and aldosterone levels. Fractionated metanephrines were normal also.

His thyroid function tests were markedly abnormal, with a very elevated free T4 at greater than $7.77 \mathrm{ng} / \mathrm{dL}$, very elevated total T3 at $360 \mathrm{ng} / \mathrm{dL}$, a markedly reduced TSH at less than $0.01 \mathrm{mcunit} / \mathrm{mL}$. This in contrast to his baseline thyroid function tests, measured the day prior to initiation of therapy, with a TSH at $2.44 \mathrm{mcunit} / \mathrm{mL}$, and a free T4 at $1.25 \mathrm{ng} / \mathrm{dL}$ (see Table). Additional tests measured the day of the event included the Thyroid Peroxidase Antibody (TPO), elevated at $574 \mathrm{IU} / \mathrm{mL}$, as well as the Thyrotropin Receptor Antibody (TRAb), elevated at $2.20 \mathrm{IU} / \mathrm{L}$. The Thyroid Stimulant Immunoglobulin (TSI), was low at less than $1.0 \mathrm{TSI}$ Index, and the Interleukin-6 levels were low at less than $5 \mathrm{pg} / \mathrm{mL}$.

The patient was successfully managed with beta-blockers; his cardiac rhythm returned to normal sinus. No corticosteroids were necessary.

Following the hyperthyroid phase, the patient became euthyroid and then hypothyroid, requiring thyroid-replacement therapy.

The use of immune-checkpoint blocking antibodies anti-cytotoxic Tlymphocyte antigen-4 (CTLA-4) and programmed cell death protein 1 (PD1), alone or in combination, have demonstrated objective antitumor responses in multiple tumor types, including melanoma, non-small cell lung cancer (NSCLC), and renal cell carcinoma (RCC). The investigational use of these agents is being increasingly applied to other tumor types.

CTLA-4 and PD-1 are two co-inhibitory receptors that are expressed on activated T-cells against which therapeutic blocking antibodies have reached routine clinical use. Immune checkpoint blockade can induce inflammatory adverse effects, termed immune-related adverse events (IRAEs), which resemble autoimmune disease.

This case is that of a middle-aged gentleman who developed a sudden but significant episode of atrial fibrillation with rapid ventricular response 21 days after his initial dose of nivolumab-ipilimumab. The patient lacked the risk factors and comorbidities that commonly coexist with atrial fibrillation, such as advanced age, alcohol consumption, family history of atrial fibrillation, hypertension, sleep apnea, and heart disease. He was found to have acute autoimmune thyroiditis, suggesting exposure to the immune checkpoint inhibitors nivolumab-ipilimumab as the causative agent.

In clinical trials, it has been determined an increased susceptibility to hypophysitis in those treated with CTLA4-targeted immunotherapy, and PD-1-targeted treatment have been predominantly linked with primary thyroid dysfunction. Additionally, the combination of nivolumab and ipilimumab has greater incidences of thyroiditis than monotherapy. These data suggest that additive effects of the two therapies might contribute to certain IRAEs.

References

Dadu R, Zobniw C, Diab A: Managing Adverse Events With Immune Checkpoint Agents. The Cancer Journal 22:121-129, 2016

Dillard T, Yedinak CG, Alumkal J, et al: Anti-CTLA-4 antibody therapy associated autoimmune hypophysitis: serious immune related adverse events across a spectrum of cancer subtypes. Pituitary 13:29-38, 2010

Abdel-Wahab N, Shah M, Suarez-Almazor ME: Adverse Events Associated with Immune Checkpoint Blockade in Patients with Cancer: A Systematic Review of Case Reports. PLOS ONE 11:e0160221, 2016

Byun DJ, Wolchok JD, Rosenberg LM, et al: Cancer immunotherapy - immune checkpoin blockade and associated endocrinopathies. Nat Rev Endocrinol 13:195-207, 2017 Kaakeh Y, Overholser BR, Lopshire JC, et al: Drug-Induced Atrial Fibrillation. Drugs 72:1617-1630, 2012

Sauter NP, Atkins MB, Mier JW, et al: Transient thyrotoxicosis and persistent hypothyroidism due to acute autoimmune thyroiditis after interleukin-2 and interferon-\&\#x3b1; therapy for metastatic carcinoma: A case report. The American Journal of Medicine 92:441-444, 1992

Gupta MK: Thyrotropin receptor antibodies: advances and importance of detection techniques in thyroid diseases. Clin Biochem 25:193-9, 1992

Heinzerling L, Ott PA, Hodi FS, et al: Cardiotoxicity associated with CTLA4 and PD1 blocking immunotherapy. Journal for ImmunoTherapy of Cancer 4:50, 2016

Naidoo J, Page DB, Li BT, et al: Toxicities of the anti-PD-1 and anti-PD-L1 immune checkpoin antibodies. Annals of Oncology 26:2375-2391, 2015

10. Brahmer JR, Lacchetti C, Thompson JA: Management of Immune-Related Adverse Events in Patients Treated With Immune Checkpoint Inhibitor Therapy: American Society of Clinical Oncology Clinical Practice Guideline Summary. Journal of Oncology Practice 14:247-249, 2018

\section{Conclusions}

In immunotherapy-related thyroiditis, hyperthyroidism is thought to be as a consequence of destruction of follicular thyroid cells with consequent liberation of thyroid hormones, but the exact mechanism is still under investigation.

Table. Thyroid function tests at baseline (i.e., the day prior to initiation of nivolumab-ipilimumab), the day of the atrial fibrillation with rapid ventricular response event (Day 0), and at 2 days, 4 days, 10 days, 13 days and 40 days following the event.

\begin{tabular}{|llllll|l|l|}
\hline $\begin{array}{l}\text { Test } \\
\text { (units) }\end{array}$ & Baseline & Day 0 & $\begin{array}{l}2 \text { days } \\
\text { post- } \\
\text { event }\end{array}$ & $\begin{array}{l}4 \text { days } \\
\text { post- } \\
\text { event }\end{array}$ & $\begin{array}{l}10 \text { days } \\
\text { post- } \\
\text { event }\end{array}$ & $\begin{array}{l}13 \text { days } \\
\text { post- } \\
\text { event }\end{array}$ & $\begin{array}{l}40 \text { days } \\
\text { post- } \\
\text { event }\end{array}$ \\
\hline $\begin{array}{l}\text { TSH } \\
\text { (mcunit/ } \\
\text { mL) }\end{array}$ & 2.44 & $<0.01$ & & & 0.09 & 1.19 & 33.91 \\
\hline $\begin{array}{l}\text { Free T4 } \\
\text { (ng/dL) }\end{array}$ & 1.25 & $>7.77$ & 4.8 & 2.69 & 1.04 & 0.76 & 0.58 \\
\hline $\begin{array}{l}\text { Total T3 } \\
\text { (ng/dL) }\end{array}$ & 360 & 239 & 176 & & & & \\
\hline
\end{tabular}

Figure. EKG the day of the atrial fibrillation with rapid ventricular response event.

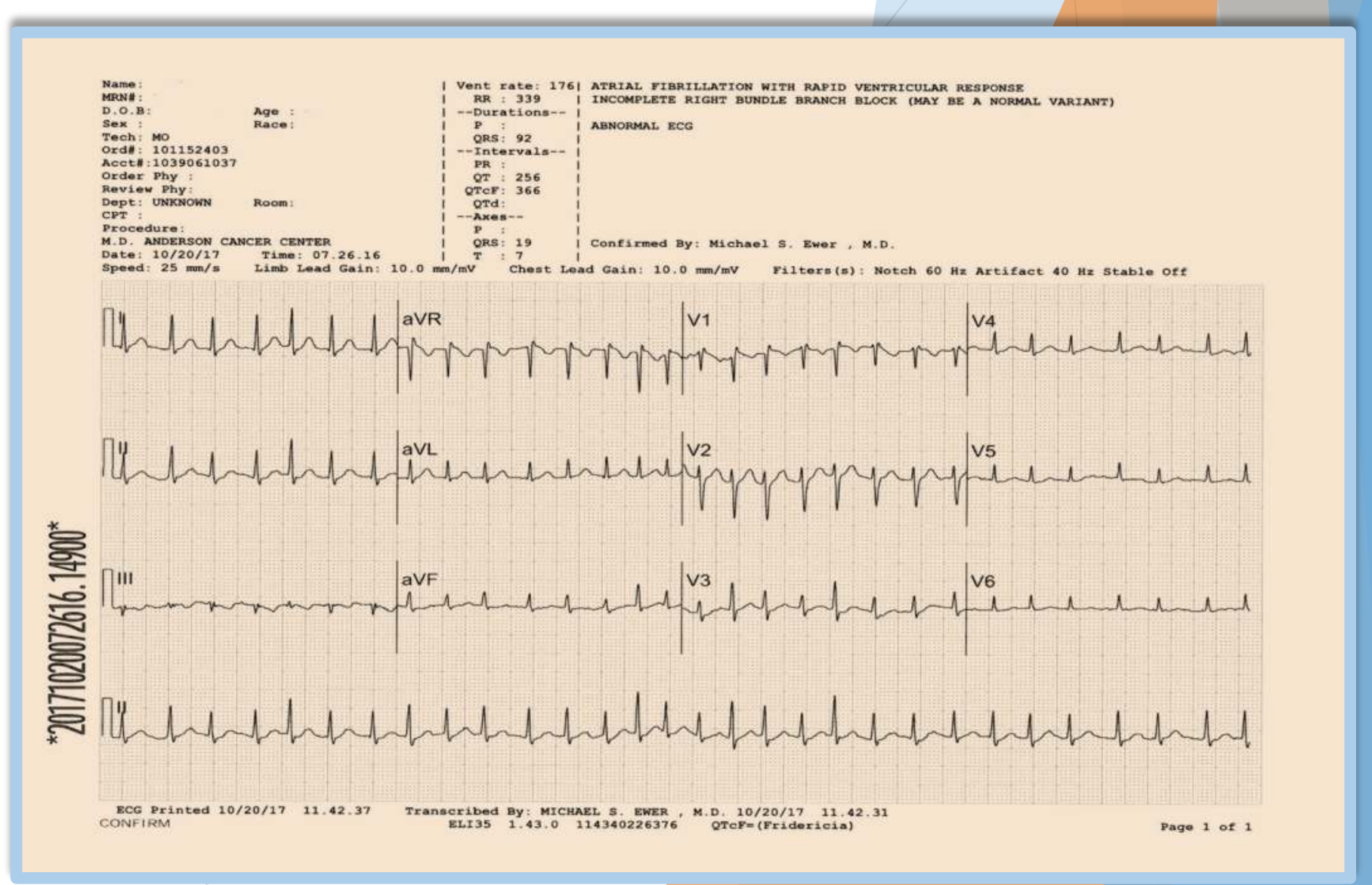

